\title{
aldehyde dehydrogenase and reactive oxygen species in nitroglycerin tolerance and cross-tolerance
}

\author{
Karsten Sydow, ${ }^{1}$ Andreas Daiber, ${ }^{1}$ Matthias Oelze, ${ }^{1}$ Zhiqiang Chen, ${ }^{2}$ Michael August, ${ }^{1}$ \\ Maria Wendt, ${ }^{1}$ Volker Ullrich, ${ }^{3}$ Alexander Mülsch, ${ }^{4}$ Eberhard Schulz, ${ }^{5}$ John F. Keaney, Jr., ${ }^{5}$ \\ Jonathan S. Stamler, ${ }^{2}$ and Thomas Münzel ${ }^{1}$ \\ ${ }^{1}$ The University Hospital Eppendorf, Division of Cardiology, Hamburg, Germany \\ ${ }^{2}$ The Howard Hughes Medical Institute, Duke University Medical Center, Durham, North Carolina, USA \\ ${ }^{3}$ Department of Biology, University Konstanz, Konstanz, Germany \\ ${ }^{4}$ Department of Physiology, University of Frankfurt, Frankfurt am Main, Germany \\ ${ }^{5}$ Boston University School of Medicine, Boston, Massachusetts, USA
}

Recent studies suggest that mitochondrial aldehyde dehydrogenase (ALDH-2) plays a central role in the process of nitroglycerin (glyceryl trinitrate, GTN) biotransformation in vivo and that its inhibition accounts for mechanism-based tolerance in vitro. The extent to which ALDH-2 contributes to GTN tolerance (impaired relaxation to GTN) and cross-tolerance (impaired endothelium-dependent relaxation) in vivo remain to be elucidated. Rats were treated for three days with GTN. Infusions were accompanied by decreases in vascular ALDH-2 activity, GTN biotransformation, and cGMP-dependent kinase (cGK-I) activity. Further, whereas in control vessels, multiple inhibitors and substrates of ALDH-2 reduced both GTN-stimulation of cGKI and GTN-induced vasodilation, these agents had little effect on tolerant vessels. A state of functional tolerance (in the GTN/cGMP pathway) was recapitulated in cultured endothelial cells by knocking down mitochondrial DNA ( $\rho^{0}$ cells). In addition, GTN increased the production of reactive oxygen species (ROS) by mitochondria, and these increases were associated with impaired relaxation to acetylcholine. Finally, antioxidants/reductants decreased mitochondrial ROS production and restored ALDH-2 activity. These observations suggest that nitrate tolerance is mediated, at least in significant part, by inhibition of vascular ALDH-2 and that mitochondrial ROS contribute to this inhibition. Thus, GTN tolerance may be viewed as a metabolic syndrome characterized by mitochondrial dysfunction.

J. Clin. Invest. 113:482-489 (2004). doi:10.1172/JCI200419267.

\section{Introduction}

The therapeutic effect of organic nitrates is due to venous and arterial dilation, which decreases myocardial oxygen consumption. It is thought that nitroglycerin (glyceryl trinitrate; GTN) induces vasorelaxation by generating $\mathrm{NO}$ or a related $S$-nitrosothiol (SNO). Both enzymatic and nonenzymatic mechanisms of

Received for publication June 23, 2003, and accepted in revised form November 4, 2003.

Address correspondence to: Thomas Münzel, Medizinische Klinik IIII, Universitäts-Krankenhaus Eppendorf, Martinistrasse 52, D-20246 Hamburg, Germany. Phone: 49-40-42803-3988; Fax: 49-40-42803-5862; E-mail: muenzel@uke.uni-hamburg.de. Karsten Sydow and Andreas Daiber contributed equally to this work.

Conflict of interest: The authors have declared that no conflict of interest exists.

Nonstandard abbreviations used: glyceryl trinitrate (GTN); $S$-nitrosothiol (SNO); mitochondrial aldehyde dehydrogenase (ALDH-2); soluble guanylyl cyclase (sGC); cGMP-dependent protein kinase I (cGK-I); reactive oxygen species (ROS); 1,2-glyceryl dinitrate (1,2-GDN); vasodilator-stimulated phosphoprotein (VASP); phosphorylated VASP at serine 239 (P-VASP); sodium nitroprusside (SNP); ebselen (Eb); porcine aortic endothelial cell (PAEC); acetylcholine (ACh).
GTN metabolism and biotransformation have been proposed. The list of candidate enzymes includes glutathione S-transferases (1), the cytochrome p450 system (2), xanthine oxidoreductase (3), and mitochondrial aldehyde dehydrogenase (ALDH-2) (4). Direct interactions of GTN with low-molecular-weight thiols may also produce vasodilator SNOs (5). NO and SNOs activate the target enzyme soluble guanylyl cyclase (sGC), increasing tissue levels of the second messenger cGMP. A cGMP-dependent protein kinase I (cGK-I) mediates vasorelaxation by phosphorylating proteins that regulate intracellular $\mathrm{Ca}^{2+}$ levels (6). Nitroglycerin can also dilate blood vessels through a cGMP-independent pathway (4).

Despite potent vasodilator effects when given acutely, the therapeutic efficacy of GTN is limited by development of tolerance and cross tolerance (impaired relaxation by other classes of nitrosovasodilators). The mechanisms underlying this time-dependent phenomenon are probably multifactorial and may involve neurohormonal counterregulatory mechanisms (so-called pseudotolerance) (7), increases in activity of the phosphodiesterase 1A1 (8), desensitization of the sGC (9), 
increases in production of reactive oxygen species (ROS) (10), and impairment of GTN biotransformation (socalled mechanism-based or classical tolerance) (4). Most recently, Chen et al. demonstrated that ALDH-2 is primarily responsible for biotransformation of GTN. They showed that ALDH-2 catalyzes the conversion of GTN to 1,2-glyceryl dinitrate (1,2-GDN) and nitrite within mitochondria (4). Inhibitors of ALDH-2 blocked the vasorelaxation by GTN that is dependent on cGMP (cGMP-independent relaxation was still evident) both in vitro and in vivo. Furthermore, treatment of vascular tissue with high concentrations of GTN resulted in both inhibition of ALDH-2 and a shift in the GTN doseresponse relationship. Thus, it appears that inhibition of ALDH-2 also underlies classical mechanism-based tolerance in vitro. The authors speculated that buildup of GTN and/or NO byproducts in mitochondria may lead to mitochondrial damage and uncoupling of respiration (4). Increased production of superoxide and other ROS would in turn oxidize critical thiols, including active-site thiols in ALDH-2 (11), further attenuating GTN-biotransformation. Superoxide also inactivates endothelium-dependent vasodilators (thereby reducing cGK-I activity). Thus, mitochondrial production of ROS would promote both mechanism-based tolerance and cross tolerance. The extent to which ALDH-2 contributes to GTN tolerance (impaired relaxation to GTN) and cross tolerance (e.g., impaired endothelium-dependent relaxation) in vivo remains to be elucidated. More specifically, whether in vivo treatment with GTN results in an inhibition of ALDH-2 and whether this predisposes to production of mitochondrial ROS remains to be determined.

Here we report that evolution of GTN tolerance in an animal model is associated with inhibition of vascular ALDH-2 activity, impairment of GTN metabolism, and increased production of ROS by mitochondria. In addition we provide data, demonstrating that increases in cGMP in response to GTN are severely blunted in cultured endothelial cells deficient in mitochondria $\left(\rho^{0}\right.$ cells). Collectively, these data provide a new paradigm for understanding both classical tolerance and cross tolerance and suggest that mitochondrial dysfunction may underlie the increased cardiovascular risk in patients taking nitrates.

\section{Methods}

Animal model for in vivo nitrate tolerance. Wistar rats treated with sham (ethanol) or NTG (treated for 3 days, 0.48 $\mu \mathrm{mol} / \mathrm{h}$ ) were studied as described recently (12).

Organ chamber experiments. Vasodilator responses to GTN were assessed in organ chambers as described (10). In selected experiments, vessels from rats treated or not treated with GTN were incubated with ALDH-2 inhibitors, including $1 \mathrm{mM}$ chloral hydrate (inhibits cytosolic [ALDH1-] and mitochondrial ALDH [ALDH-2]) and $10 \mu \mathrm{M}$ daidzin (specific for ALDH-2) (13), and substrates, $1 \mathrm{mM}$ acetaldehyde and $1 \mu \mathrm{M}$ benomyl, an irreversible inhibitor of ALDH (14). $c$ GK-I activity. Aortic segments $(1 \mathrm{~cm})$ from GTN and sham-treated rats were frozen and homogenized in liquid nitrogen. SDS-PAGE electrophoresis and electroblotting were performed as described $(15,16)$. Immunoblotting was performed with a polyclonal $\mathrm{Ab}$ against cGK-I and a mouse mAb (16C2) specific for phosphorylation of vasodilator-stimulated phosphoprotein at serine 239 (P-VASP), as described $(15,16)$. The effect of benomyl $(1 \mu \mathrm{M})$ on P-VASP was tested in the presence of either GTN $(0.1 \mu \mathrm{M})$ or sodium nitroprusside (SNP) $(0.1 \mu \mathrm{M})$.

GTN biotransformation. The conversion of ${ }^{14} \mathrm{C}$-labeled GTN into 1,2-GDN and 1,3-GDN was measured by TLC and liquid scintillation spectrometry as described by Brien et al. (17) and Chen et al. (4).

$A L D H$ activity in isolated rat heart mitochondria and vasculartissue (cytosol vs. mitochondria). The activity of ALDH in intact blood vessels and isolated mitochondria was determined by measuring the conversion of benzaldehyde to benzoic acid (18) and/or the conversion of propionaldehyde to propionic acid. Briefly, aortic rings (1 $\mathrm{cm}$ ) from sham or GTN-treated rats were incubated for 30 minutes at $37^{\circ} \mathrm{C}$ in $0.25 \mathrm{ml}$ Krebs-HEPES buffer with or without benomyl $(10 \mu \mathrm{M})$. Benzaldehyde $(200 \mu \mathrm{M})$ was then added $\left(60\right.$ minutes at $\left.37^{\circ} \mathrm{C}\right)$. Aortic rings were homogenized on ice and centrifuged at $16,000 \mathrm{~g}$ (30 minutes at $4^{\circ} \mathrm{C}$ ). The supernatant was purified by size-exclusion centrifugation in a Microcon YM-10 filter device (Millipore Corp., Bedford, Massachusetts, USA). Samples were stored at $-80^{\circ} \mathrm{C}$.

Alternatively, in a complementary approach, deepfrozen rat aortic rings were homogenized for 30 seconds in a liquid nitrogen precooled dismembranator (B. Braun Biotech International $\mathrm{GmbH}$, Melsungen, Germany). The powdered tissue was dispersed in 5 vol icecold aqueous $30 \mathrm{mM}$ potassium phosphate buffer (deoxygenated with nitrogen gas), $\mathrm{pH} 7.5$, vortexed, sonicated, and centrifuged at $10,000 \mathrm{~g}$ for 10 minutes. ALDH activity was assessed at room temperature by monitoring NADH formation from $\mathrm{NAD}^{+}$at $340 \mathrm{~nm}$ in an Uvikon 941 dual-beam photospectrometer. The assay mixture $(0.5 \mathrm{ml})$ contained $100 \mathrm{mM}$ Tris- $\mathrm{HCl}(\mathrm{pH}$ 8.5), 1 mM NAD ${ }^{+}, 1 \mathrm{mM} 4$-methylpyrazole, and $100 \mu \mathrm{g}$ protein. The reaction was started by addition of $1 \mathrm{mM}$ propionaldehyde to the cuvette, and absorbance changes were recorded for 10 minutes. The mean rate of absorbance change was taken as a measure of ALDH activity $\left(0.0125 \mathrm{~A}_{340}\right.$ was equivalent to $\left.1 \mathrm{nmol} / \mathrm{mg} / \mathrm{min}\right)$.

Rat heart mitochondria was prepared according to a modified method of Robinson et al. (19). Hearts from sham or GTN-treated rats were homogenized in HEPES buffer and centrifuged at $1,500 \mathrm{~g}\left(10\right.$ minutes at $\left.4^{\circ} \mathrm{C}\right)$ and 2,000 $\mathrm{g}$ for 5 minutes (the pellets were discarded). The supernatant was then centrifuged at $16,000 \mathrm{~g}$ for 20 minutes, and the pellet was resuspended in $1 \mathrm{ml}$ of HEPES buffer. The latter step was repeated, and the pellet was suspended in $1 \mathrm{ml}$ of Tris buffer. The mitochondrial fraction (total protein approximately 3-5 $\mathrm{mg} / \mathrm{ml}$ ) was kept on ice and diluted to approximately 
$1 \mathrm{mg} / \mathrm{ml}$ protein in $0.25 \mathrm{ml}$ of 3-morpholinopropanesulfonic acid (MOPS) buffer in the presence or absence of ALDH-2 inhibitors ( $10 \mu \mathrm{M}$ benomyl, 40 $\mu \mathrm{M}$ daidzin, $500 \mu \mathrm{M}$ GTN), antioxidants ( $2 \mathrm{mM} \mathrm{DTT}$ ) or $1 \mathrm{mM}$ uric acid or $20 \mu \mathrm{g} / \mathrm{ml}$ antimycin A (the inhibitor of mitochondrial complex III), plus $4 \mathrm{mM}$ succinate for 30 minutes at $37^{\circ} \mathrm{C}$. Incubations with benzaldehyde $(200 \mu \mathrm{M})$ were for an additional 60 minutes at $37^{\circ} \mathrm{C}$. Samples were sonicated, centrifuged at $20,000 \mathrm{~g}\left(4^{\circ} \mathrm{C}\right)$ for 20 minutes, and the supernatant was purified by size exclusion centrifugation in a Microcon YM-3 filter device.

To determine the effects of in vivo GTN infusion on vascular ALDH, six aortas from GTN or sham-treated rats were pooled together. The activity of ALDH-2 was measured as described above. In addition, the cytosolic fraction was prepared by an additional centrifugation step at $100,000 \mathrm{~g}$ for 60 minutes at $4^{\circ} \mathrm{C}$. The activity of cytosolic ALDH was quantified after incubation of the supernatant $(2 \mathrm{mg} / \mathrm{ml}$ total protein) in PBS with 200 $\mu \mathrm{M} \mathrm{NAD}^{+}$and benzaldehyde for 30 minutes at $37^{\circ} \mathrm{C}$. The samples were purified and measured by HPLC as described below. The effects of benomyl $(1-100 \mu \mathrm{M})$, chloral hydrate $(0.1-10 \mathrm{mM})$, and daidzin $(1 \mu \mathrm{M}-1 \mathrm{mM})$ on mitochondrial and cytosolic ALDH were tested.

HPLC. One hundred microliters of each sample were subjected to HPLC analysis. The system consisted of a LKB pump, a Knaur UV/Vis detector, and a $\mathrm{C}_{18}$-Nucleosil $125 \times 4$ 100-3 reversed-phase column from Macherey-Nagel GmbH Co. KB (Düren, Germany). The mobile phase contained acetonitrile $(25 \mathrm{vol} / \mathrm{vol}$ by percentage) in $50 \mathrm{mM}$ citric acid buffer $(75 \mathrm{vol} / \mathrm{vol}$ by percentage), $\mathrm{pH}$ 2.2. The substrate and its products were isocratically eluted at a flow rate of $0.8 \mathrm{ml} / \mathrm{min}$, detected at either 265 or $275 \mathrm{~nm}$, and quantified using internal and external standards (benzyl alcohol, benzoic acid, and benzaldehyde). The typical retention times were 5.1, 7.3, and 10.5 minutes, respectively.

Measurement of ROS production from isolated heart mitochondria. Mitochondrial stock solutions were diluted to final total protein concentrations of approximately 0.1 $\mathrm{mg} / \mathrm{ml}$ in $0.5 \mathrm{ml}$ of MOPS buffer. The dye L-012 (100 $\mu \mathrm{M})$ was used as described $(20,21)$ to quantify ROS following addition of succinate ( $4 \mathrm{mM}$ final concentration). Chemiluminescence was monitored over 5 minutes using a Lumat LB9507 from Berthold Technologies (Wildbad, Germany), and the signal at 5 minutes was expressed as counts per minute. ROS production was quantified in mitochondria from GTN-treated animals (in vivo) and in mitochondria exposed to increasing concentrations of GTN in vitro. In selected experiments, mitochondria were preincubated with DTT $(100 \mu \mathrm{M})$ or the reductant/antioxidants uric acid (20 $\mu \mathrm{M})$ and ebselen $(\mathrm{Eb} ; 100 \mu \mathrm{M})$. In addition, mitochondrial ROS formation was quantified after inhibition of the respiratory chain with antimycin $\mathrm{A}(20 \mu \mathrm{g} / \mathrm{ml})$ and stimulation with succinate $(4 \mathrm{mM})$.

Cell culture experiments. Porcine aortic endothelial cells (PAECs) were harvested and cultured in M199 medium

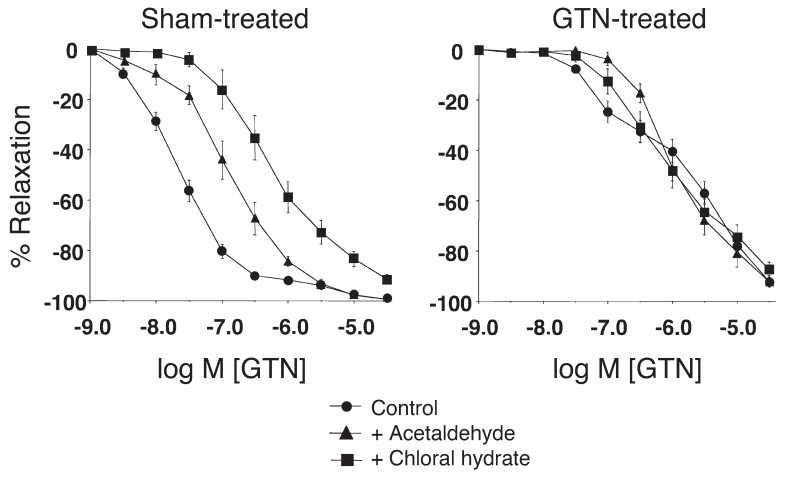

Figure 1

Effects of inhibition of ALDH-2 on GTN-induced relaxation of rat aorta from sham-treated (a) and GTN-treated rats (b). The inhibitory effects of ALDH-2 inhibitor and substrate $(1 \mathrm{mM}$ chloral hydrate, squares, and $1 \mathrm{mM}$ acetaldehyde, triangles, respectively) were markedly attenuated in vessels from GTN-treated animals, identifying the desensitization with ALDH-2. Data are mean \pm SEM from 6-30 independent experiments. [GTN], concentration of GTN.

supplemented with $20 \% \mathrm{FBS}, 50 \mu \mathrm{g} / \mathrm{ml}$ heparin, 100 $\mathrm{U} / \mathrm{ml}$ penicillin, $100 \mu \mathrm{g} / \mathrm{ml}$ streptomycin, and $2 \mathrm{mM}$ L-glutamine. Confluent cells between passages 3 and 8 were used in experiments. To inhibit mitochondrial gene transcription and activity, $50 \mathrm{ng} / \mathrm{ml}$ ethidium bromide was added to the regular medium. As alternative energy and nucleotide sources, cells were supplied with $110 \mathrm{mg} / \mathrm{ml}$ pyruvate and $50 \mu \mathrm{g} / \mathrm{ml}$ uridine as described (22). Western blot analysis for cytochrome $c$ oxidase subunit I (Molecular Probes Inc., Leiden, The Netherlands) was performed to assess mitochondrial gene expression. Incubation of PAECs with ethidium, pyruvate, and uridine for 5 days completely depleted cellular protein levels of cytochrome $c$ oxidase I, a protein that is encoded only by mitochondrial DNA. This observation is an established criteria for inhibition of mitochondrial function (23).

Determination of cellular cGMP levels. Confluent PAECs in six-well plates were washed two times with Krebs/HEPES buffer $(124 \mathrm{mM} \mathrm{NaCl}, 5 \mathrm{mM} \mathrm{KCl}, 1 \mathrm{mM} \mathrm{MgCl}, 1.5 \mathrm{mM}$ $\mathrm{CaCl}_{2}, 0.16 \mathrm{mM} \mathrm{HPO}_{4}, 0.4 \mathrm{mM} \mathrm{H}_{2} \mathrm{PO}_{4}, 5 \mathrm{mM} \mathrm{NaHCO}_{3}$, $5.6 \mathrm{mM}$ D-glucose, and $22 \mathrm{mM}$ HEPES, $\mathrm{pH}$ 7.35) and equilibrated for 30 minutes in Krebs/HEPES buffer containing the phosphodiesterase inhibitor 3-isobutyl-1methylxanthine $(250 \mu \mathrm{M})$ and L-arginine $(200 \mu \mathrm{M})$. Cells were then treated with GTN for 2 minutes at concentrations between $100 \mathrm{nM}$ and $100 \mu \mathrm{M}$. The reaction was stopped by addition of $6 \%$ ice-cold trichloroacetic acid and cellular cGMP determined as described (24).

Statistical analysis. Results are expressed as mean plus or minus SEM. One-way ANOVA was used for comparisons of L-012 chemiluminescence, cGK-I activity, cGMP responses, and ALDH-2 activity. The $\mathrm{ED}_{50}$ value for each experiment was obtained by log transformation. Comparisons of vascular responses were performed using ANOVA. A Scheffé post hoc test was used to examine differences between groups. Spearman's 


\section{Table 1}

Effects of GTN treatment on endothelial function and of ALDH-2 inhibitors on GTN relaxations of aortas with and without GTN treatment

\begin{tabular}{|c|c|c|c|c|}
\hline & \multicolumn{2}{|c|}{$\begin{array}{c}\text { Potency } \\
\left(\mathrm{ED}_{50},{ }^{\mathrm{A}}-\log \mathrm{M}\right)\end{array}$} & \multicolumn{2}{|c|}{$\begin{array}{l}\text { Efficacy (maximal } \\
\text { relaxation, } \% \text { ) }\end{array}$} \\
\hline & GTN & $\mathrm{ACh}$ & GTN & $\mathrm{ACh}$ \\
\hline Sham-treated & $-7.63 \pm 0.07$ & $-7.36 \pm 0.07$ & $99 \pm 1$ & $90 \pm 1$ \\
\hline + Acetaldehyde & $-6.88 \pm 0.12^{B}$ & & $100 \pm 1$ & \\
\hline + Chloral hydrate & $-6.32 \pm 0.15^{\mathrm{B}}$ & & $92 \pm 2^{B}$ & \\
\hline + Benomyl & $-6.69 \pm 0.10^{B}$ & & $99 \pm 1$ & \\
\hline + Daidzin & $-6.51 \pm 0.29^{B}$ & & $98 \pm 1$ & \\
\hline GTN-treated & $-6.00 \pm 0.14^{B}$ & $-7.10 \pm 0.19$ & $92 \pm 2^{B}$ & $75 \pm 3^{B}$ \\
\hline + Acetaldehyde & $-5.92 \pm 0.14^{B}$ & & $92 \pm 3^{B}$ & \\
\hline + Chloral hydrate & $-6.13 \pm 0.14^{B}$ & & $87 \pm 3^{B}$ & \\
\hline
\end{tabular}

Each value is the mean \pm SEM of 5-33 experiments. ${ }^{A} E D_{50}$ are concentrations that produced $50 \%$ of maximal relaxation to each drug. ${ }^{\mathrm{B}} P<0.05$ versus sham-treated rats.

rank correlation coefficients were calculated to evaluate relationships between variables. $P$ values less than 0.05 were considered to be significant.

\section{Results}

Organ chamber experiments. In vitro incubation of native vessels from sham-treated rats with either the ALDH inhibitor chloral hydrate, the specific ALDH-2 inhibitor daidzin $(10 \mu \mathrm{M})$, or the ALDH substrate acetaldehyde (1 $\mathrm{mM}$ ) markedly shifted the GTN dose-response curve to the right (Figure 1; Table 1). Similarly, GTN treatment for 3 days markedly decreased the sensitivity of the vasculature to GTN. Incubation of tolerant vessels with ALDH-2 inhibitors, however, did not produce a further shift in the GTN dose response (Figure 1; Table 1).

Incubation of control tissue with chloral hydrate and acetaldehyde did not modify endothelium-dependent relaxation to acetylcholine (ACh). In contrast, in vivo treatment with GTN caused a significant degree of endothelial dysfunction, as indicated by a significant shift in the ACh dose-response relationship to the right (Table 1).

Effect of ALDH-2 inbibition on cGK-I activity and GTNdependent relaxation. Incubation of vascular tissue with GTN $(0.1 \mu \mathrm{M})$ increased P-VASP in aortic rings by more than $80 \%$ (Figure 2). The GTN-mediated increase in cGK-I activity was prevented by the ALDH-2 inhibitor benomyl $(1 \mu \mathrm{M})$. Inhibition of GTN-dependent VASP phosphorylation was associated with a marked desensitization of the vessels to GTN and a significant rightward shift in the dose-response relationship (Table 1). Exposure of aortic rings to SNP $(0.1 \mu \mathrm{M})$ resulted in activation of cGK-I, which was not modified by benomyl (Figure 2). Likewise, SNP-induced relaxations were not affected by benomyl (data not shown).

Inhibition of vascular ALDH-2 with benomyl had no significant effect on basal vascular cGK-I activity. By contrast, in vivo treatment with GTN for 3 days reduced cGK-I activity about 50\% (control: $100 \% \pm 6 \%$; GTN-treated: $51 \% \pm 4 \%$ ).
Vascular and mitochondrial ALDH activity. Incubation of isolated aortic rings with benomyl $(10 \mu \mathrm{M})$ decreased vascular ALDH activity approximately $80 \%$, as measured by conversion of benzaldehyde to benzoic acid (Figure 3). The specific, albeit relatively insoluble inhibitor of ALDH-2, daidzin $(100 \mu \mathrm{M})$, decreased vascular ALDH activity approximately $50 \%$ (not shown). In vessels from rats treated with GTN in vivo, total ALDH activity was reduced more than 50\% compared with the activity in vessels from sham-treated controls (Figure 3a).

Addition of propionaldehyde to reaction mixtures produced a linear increase in NADH formation over 10 minutes. The mean ALDH activity in homogenates from control aortas was $2.6 \pm 0.9 \mathrm{nmol} / \mathrm{mg} / \mathrm{min}$, whereas ALDH activity in tolerant aorta averaged $1.2 \pm 0.3$ $\mathrm{nmol} / \mathrm{mg} / \mathrm{min}(P<0.05 ; n=5)$.

ALDH activity in isolated rat heart mitochondria was markedly inhibited by in vitro treatment with benomyl $(10 \mu \mathrm{M})$ or GTN $(500 \mu \mathrm{M})$ and by in vivo treatment with GTN (Figure 3b). Incubation of mitochondria from tolerant animals with DTT $(2 \mathrm{mM})$ normalized ALDH activity. A significant ( $>50 \%$ ) decrease in ALDH-2 activity was also observed when ROS formation was stimulated in isolated mitochondria by the complex III inhibitor antimycin A (not shown).

Whereas benomyl $(10 \mu \mathrm{M})$ inhibited the ALDH-2 in isolated mitochondria from rat heart by more than $90 \%$, it also inhibited the cytosolic isoform of ALDH in cardiac homogenates by more than $85 \%$. In contrast, 10 $\mu \mathrm{M}$ daidzin inhibited ALDH-2 activity more than $40 \%$, while cytosolic ALDH remained unchanged.

Similar results were obtained in aortic mitochondria, where ALDH-2 activity was reduced from $31.5 \pm 4.6$ to $18.6 \pm 4.2 \mu \mathrm{M} / \mathrm{mg}(n=3)$ in tolerant tissue. That is, in

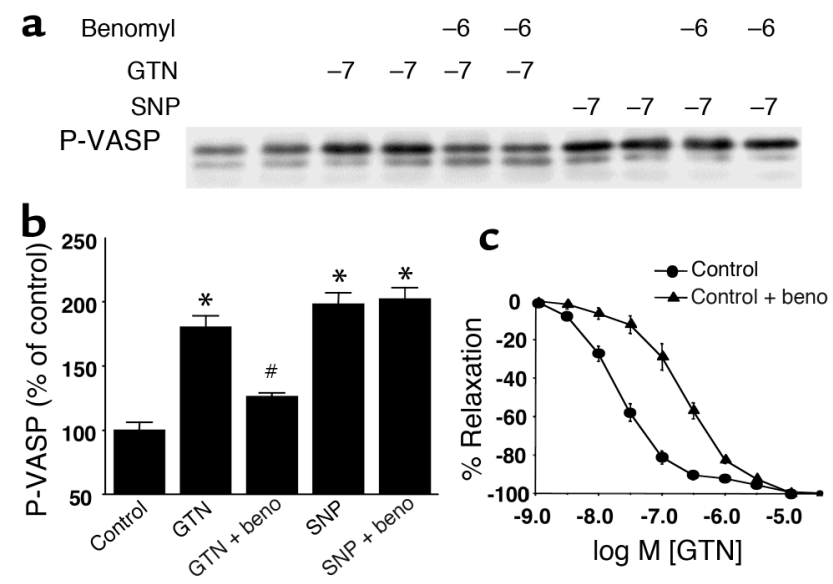

\section{Figure 2}

Effects of ALDH-2 inhibition on cGK-I activity in rat aorta. Degree of VASP phosphorylation (P-VASP) and vasorelaxation by GTN and SNP in the presence and absence of the ALDH-2 inhibitor benomyl (beno) $(1 \mu \mathrm{M})$. Benomyl attenuated GTN-induced $(0.1 \mu \mathrm{M})$ but not SNP-induced $(0.1 \mu \mathrm{M})$ activation of cGK-I. (a) Original Western blot; (b) densitometric quantification; (c) benomyl-induced inhibition of GTN-induced vasodilation (triangle). Data are mean \pm SEM from three to five experiments. ${ }^{*} P<0.05$ vs. control; ${ }^{\#} P<0.05$ vs. GTN. 

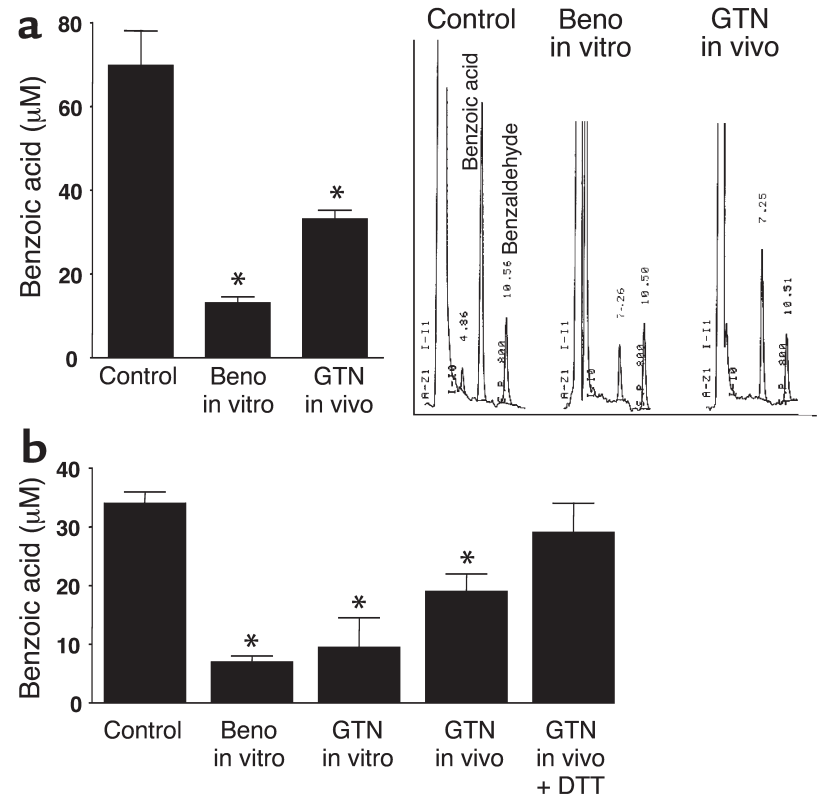

Figure 3

Effects of GTN-treatment on vascular (a) and mitochondrial (b) ALDH (as assessed by the conversion of benzaldehyde into benzoic acid). The substrate and product were identified and quantified by HPLC analysis. (a) Mean \pm SEM from three separate experiments (left-hand side). Right-hand side shows three representative chromatograms. Both GTN infusion (3 days) and in vitro exposure inhibits vascular ALDH. (b) In vivo and in vitro inhibition of ALDH-2 by GTN and protection by DTT. Data are mean \pm SEM from six to eight separate experiments. ${ }^{*} P<0.05$ vs. control.

vivo tolerance resulted in an approximately $40 \%$ loss of vascular ALDH-2 activity.

Vascular GTN biotransformation. The total GTNmetabolizing activity was decreased in tolerant vessels $(123.5 \mathrm{pmol} / \mathrm{min} / \mathrm{g}$ tissue for control tissue versus $90.6 \mathrm{pmol} / \mathrm{min} / \mathrm{g}$ tissue for the tolerant tissue). GTN inhibited ALDH-catalyzed 1,2-GDN formation by slightly more than $50 \%$ (Figure 4); 1,2-GDN formation for control rats was $77.8 \pm 9.2 \mathrm{pmol} / \mathrm{min} / \mathrm{g}$ tissue and $36.0 \pm 3.4 \mathrm{pmol} / \mathrm{min} / \mathrm{g}$ tissue for GTN-treated rats $(P<0.001)$ (Figure 4$)$. The formation of the nonspecific 1,3 -GDN metabolite increased $(45.7 \pm 5.8$ versus $54.6 \pm 5.8 \mathrm{pmol} / \mathrm{min} / \mathrm{g}$ tissue; $P=0.04)$ after GTN treatment (Figure 4).

Mitochondrial ROS production. Formation of ROS in heart mitochondria from rats treated with NTG for 3 days was increased approximately $50 \%$ compared with controls (Figure 5). Furthermore, incubation of isolated mitochondria from control animals with GTN (5 and $50 \mu \mathrm{M})$ caused a dose-dependent increase in ROS production (Figure 5). Uric acid $(20 \mu \mathrm{M})$ almost completely inhibited the L-012-enhanced chemiluminescence derived from GTN $(50 \mu \mathrm{M})$. DTT and Eb (each $100 \mu \mathrm{M}$ ) also entirely prevented the increase in ROS generated by in vitro incubation with $5 \mathrm{mM} \mathrm{GTN}$ (not shown). Mitochondrial ROS production was increased 100 -fold upon inhibition of complex III with antimycin $A$ in the presence of succinate (not shown).
Mitochondrial role in GTN-induced cGMP production. To confirm the role of functioning mitochondria in GTN bioactivity, we inhibited mitochondrial gene transcription and protein synthesis with ethidium bromide (Figure 6). Inhibition of mitochondrial function in this manner was associated with a marked reduction in GTN-induced cGMP production (Figure 6).

\section{Discussion}

The present studies demonstrate that in vivo treatment with GTN leads to (a) reduced GTN biotransformation by mitochondrial ALDH; (b) increased mitochondrial production of ROS; (c) attenuated cGK-I activity; and (d) endothelial dysfunction. Inhibition of mitochondrial ALDH and increased mitochondrial ROS are thus biochemical correlates of tolerance and cross tolerance, respectively. We also show that GTN-mediated increases in cGMP depend on functioning mitochondria. Agents that preserve ALDH-2 activity and/or reduce mitochondrial ROS production may thus ameliorate tolerance. Collectively, these findings suggest that mitochondrial dysfunction plays a key role in evolution of nitrate tolerance.

Nitrates are widely used to treat coronary artery disease and congestive heart failure. Despite acute anti-ischemic and vasodilator properties, the longterm efficacy of these compounds is questionable. Tolerance occurs within 1-3 days of continuous treatment in patients with acute myocardial infarction (25), chronic ischemic heart disease, and chronic congestive heart failure (26). Chronic treatment also leads to endothelial dysfunction (cross tolerance) $(27,28)$, a direct predictor of cardiovascular morbidity and mortality (29). Although the precise mechanisms remain poorly defined, there is growing evidence that impairment of GTN biotransformation and/or increased ROS production contribute to tolerance and cross tolerance.

Recent studies identify ALDH-2 with GTN metabolism and tolerance (4). Conclusions were based on

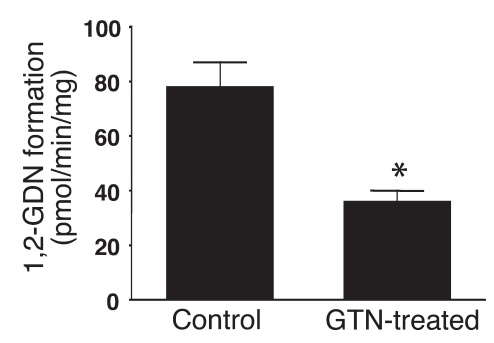

\section{Figure 4}

Effects of in vivo GTN treatment on GTN metabolism as assessed by the conversion of ${ }^{14} \mathrm{C}$-labeled GTN into 1,2-GDN. The enzymatic metabolite is significantly decreased in vessels from tolerant animals. Three rat thoracic aortas were cut into small pieces and incubated with $1 \mu \mathrm{M}{ }^{14} \mathrm{C}$-labeled GTN for 2 minutes at $37^{\circ} \mathrm{C}$ (Krebs buffer). GTN and its metabolites were separated and quantified as described in Methods. Data are mean \pm SEM from six separate experiments. ${ }^{*} P<0.05$ vs. control. 


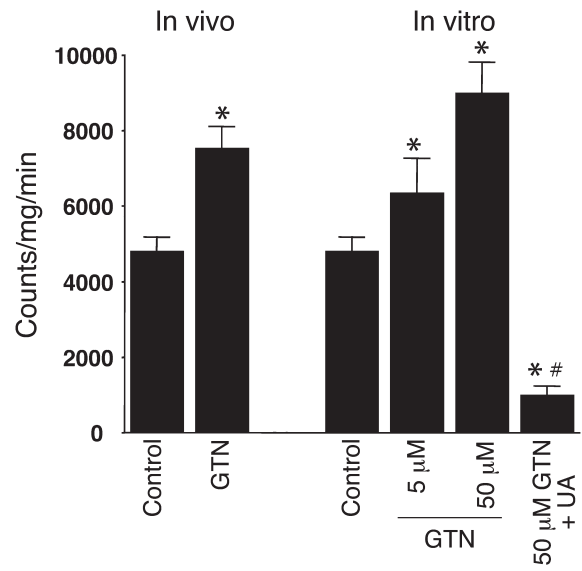

Figure 5

Effects of in vivo and in vitro GTN-treatment on mitochondrial production of ROS as assessed by L-012 enhanced chemiluminescence. Data are mean \pm SEM from 4-19 separate experiments. UA, uric acid. ${ }^{*} P<0.05$ vs. control; ${ }^{\#} P<0.05$ vs. $50 \mu \mathrm{M}$ GTN.

experiments in vitro and in vivo showing that inhibitors and a substrate of ALDH-2 (cyanamide, acetaldehyde, and chloral hydrate) induced a marked shift of the GTN dose-response relationship to the right, while relaxations to the NO donor SNP were unaltered (4). Further, inhibition of ALDH-2 significantly reduced vascular cGMP levels in response to acute GTN challenge. It was also shown that toleranceinducing concentrations of GTN in vitro resulted in an inhibition of ALDH-2 activity and an inhibition of GTN biotransformation (1,2-GDN formation). But whether similar mechanisms underlie tolerance in vivo remained untested.

Using isolated rat aortic rings, we demonstrated a marked attenuation of the GTN vasodilator potency following incubation with acetaldehyde and choral hydrate, as previously observed in rabbit aortic rings, as well as with daidzin, a specific ALDH-2 inhibitor. In addition, activation of cGK-I (as assessed by the phosphorylation status of the VASP at serine 239; ref. 30) and vasodilation by GTN were markedly inhibited by the ALDH-2 inhibitor benomyl $(1 \mu \mathrm{M})$, whereas benomyl did not modify SNP-induced phosphorylation of VASP or vasorelaxation. These results point to a specific role of ALDH-2 in the cGMP-cGK-I mechanism of GTN vasorelaxation.

Treatment of rats for 3 days with GTN resulted in a significant degree of tolerance as measured by decreased sensitivity of vascular tissue to GTN. Notably, in vitro treatment of tolerant rings with ALDH-2 inhibitors did not cause a further shift in the GTN dose-response curve. These observations are well rationalized by inhibition of ALDH-2, which was confirmed in vascular tissues directly (using two methods) and in isolated mitochondria from aorta and heart. We also showed that in vivo treatment with GTN or exposure to benomyl reduced GTN biotransformation (1,2-GDN formation).
Many of our studies were done with benomyl, a relatively nonspecific ALDH inhibitor (14). We obtained similar results with acetaldehyde, however, the preferred ALDH-2 substrate, and we verified that daidzin, which selectively inhibits ALDH-2 (13) $(\sim 50 \%$ loss of vascular ALDH activity in our studies) also abrogated GTN relaxations. Further, Chen et al. previously demonstrated that mouse macrophage homogenates, deficient in mitochondria, have no GTN-metabolizing capacity. We have now extended these observations to a more relevant cell type and physiological system by creating endothelial cells deficient in functioning mitochondria (so-called $\rho^{0}$ cells) (22) (Figure 6). GTN-stimulated increases in cGMP were markedly attenuated in the absence of functioning mitochondria. Collectively, these data provide strong support for the proposition that ALDH-2 contributes to GTN bioactivation and that inhibition of this enzyme contributes to in vivo tolerance. It will be interesting to learn whether the well-known differences in the sensitivity of different vessels (arteries, veins, arterioles) to GTN is related to differences in the activity or expression of ALDH-2.

A major drawback of chronic GTN treatment in humans is cross tolerance to endothelium-dependent vasodilators (31); ACh responses ex vivo (10) and in vivo - across forearm $(27,28)$, coronary conductance (32), and resistance vessels - are impaired. We and others have postulated that GTN treatment stimulates the production of superoxide (10,33-35), which is known to impair endothelium-dependent relaxation and activation of cGK-I. Early studies by Needleman and Hunter showed that incubation of isolated heart mitochondria with high concentrations of

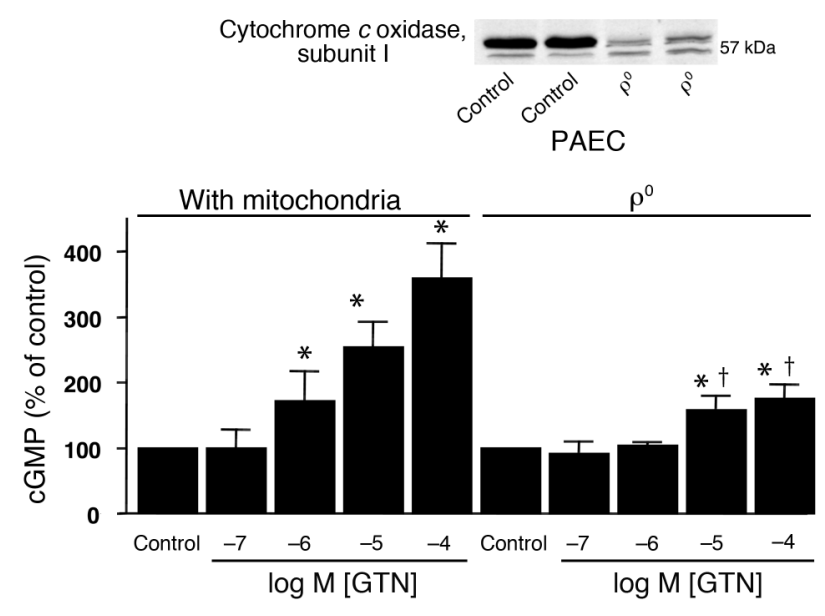

Figure 6

Effects of GTN on cGMP responses of cultured PAECs with and without mitochondria ( $\rho^{0}$ cells). Increases in cGMP were markedly suppressed in cells without mitochondria. The insert at the top righthand side shows a Western blot analysis for cytochrome $c$ oxidase (in $\rho^{0}$ cells, the expression is drastically reduced). Data are mean \pm SEM from three to four separate experiments. ${ }^{*} P<0.05$ vs. control; $\dagger P<0.05$ vs. cells with mitochondria. 
nitrates stimulated oxygen consumption and uncoupled oxidative phosphorylation $(36,37)$, observations consistent with a mitochondrial source of ROS. Regardless of the exact mechanism by which GTN stimulates ROS production (e.g., through direct damage to mitochondria, blockade of respiration by NO, and/or a "diaphorase-like" activity), loss of ALDH-2 activity should cause GTN to accumulate in mitochondria and thus amplify the effect. Indeed, we now demonstrate that GTN treatment in vitro and in vivo decreases the activity of mitochondrial ALDH in aorta and heart, and simultaneously increases mitochondrial ROS production.

Chen et al. proposed that oxidation of essential thiol groups in the active site of the enzyme underlies the molecular basis of tolerance, an observation supported by the results of the present studies. It is not known to what degree substrate GTN or ROS or both mediate ALDH-2 inactivation, however. In studies with purified yeast ALDH, we find that GTN, superoxide, and peroxynitrite (the product of $\mathrm{NO}$ /superoxide interaction) are all capable of directly inhibiting the enzyme (unpublished observations). We have also shown that mitochondrial ROS formation induced by respiratory chain blockade with antimycin $\mathrm{A}$ is associated with a loss of more than $50 \%$ of ALDH-2 activity. These observations support the idea that oxidative stress may contribute directly to mechanism-based tolerance, either by oxidative inhibition of ALDH-2 or perhaps by oxidizing key enzyme cofactors. Irrespective of the exact sequence of events, incubation of tolerant tissue with various reductants and antioxidants completely restored vascular ALDH activity and simultaneously normalized mitochondrial ROS production. Inasmuch as nitrate tolerance may underlie the increases in cardiac morbidity seen with chronic nitrate use (38), these observations may have therapeutic implications.

More generally, mitochondrial injury and the ensuing oxidative stress unify concepts of tolerance and cross tolerance and provide a molecular rationale for the range of agents that seemingly prevent the development of nitrate tolerance (for review see ref. 7). It is likely that agents ameliorating oxidative stress (e.g., angiotensin-converting enzyme inhibitors, AT1 receptor blockers, L-arginine, ascorbate) will restore sensitivity to NO donors, but it remains to be seen whether they are able to prevent inhibition of ALDH-2 during prolonged GTN treatment. Likewise, it will be of interest to see whether ebselen and uric acid, which lessen mitochondrial ROS production and preserve ALDH-2 activity in vitro, can also do so in vivo and whether this confers protection from tolerance.

\section{Acknowledgments}

We thank Claudia Kuper and Hartwig Wieboldt for expert technical assistance. Thomas Münzel is a recipient of a grant from the Deutsche Forschungsgemeinschaft Mu (1079 4-1).
1. Lau, D.T., Chan, E.K., and Benet, L.Z. 1992. Glutathione S-transferasemediated metabolism of glyceryl trinitrate in subcellular fractions of bovine coronary arteries. Pharm. Res. 9:1460-1464.

2. McDonald, B.J., and Bennett, B.M. 1993. Biotransformation of glyceryl trinitrate by rat aortic cytochrome P450. Biochem. Pharmacol. 45:268-270.

3. O'Byrne, S., et al. 2000. Inhibition of platelet aggregation with glyceryl trinitrate and xanthine oxidoreductase. J. Pharmacol. Exp. Ther. 292:326-330.

4. Chen, Z., Zhang, J., and Stamler, J.S. 2002. Identification of the enzymatic mechanism of nitroglycerin bioactivation. Proc. Natl. Acad. Sci. U. S. A. 99:8306-8311.

5. Feelisch, M., and Kelm, M. 1991. Biotransformation of organic nitrates to nitric oxide by vascular smooth muscle and endothelial cells. Biochem. Biophys. Res. Commun. 180:286-293.

6. Lincoln, T.M., Dey, N., and Sellak, H. 2001. Invited review: cGMP-dependent protein kinase signaling mechanisms in smooth muscle: from the regulation of tone to gene expression. J. Appl. Physiol. 91:1421-1430.

7. Gori, T., and Parker, J.D. 2002. The puzzle of nitrate tolerance: pieces smaller than we thought? Circulation. 106:2404-2408.

8. Kim, D., et al. 2001. Upregulation of phosphodiesterase 1A1 expression is associated with the development of nitrate tolerance. Circulation. 104:2338-2343.

9. Artz, J.D., Schmidt, B., McCracken, J.L., and Marletta, M.A. 2002. Effects of nitroglycerin on soluble guanylate cyclase: implications for nitrate tolerance. J. Biol. Chem. 277:18253-18256.

10. Munzel, T., Sayegh, H., Freeman, B.A., Tarpey, M.M., and Harrison, D.G. 1995. Evidence for enhanced vascular superoxide anion production in nitrate tolerance. A novel mechanism underlying tolerance and cross-tolerance. J. Clin. Invest. 95:187-194.

11. Steinmetz, C.G., Xie, P., Weiner, H., and Hurley, T.D. 1997. Structure of mitochondrial aldehyde dehydrogenase: the genetic component of ethanol aversion. Structure. 5:701-711.

12. Munzel, T., et al. 2000. Effects of long-term nitroglycerin treatment on endothelial nitric oxide synthase (NOS III) gene expression, NOS IIImediated superoxide production, and vascular NO bioavailability. Circ. Res. 86:E7-E12.

13. Keung, W.M., Klyosov, A.A., and Vallee, B.L. 1997. Daidzin inhibits mitochondrial aldehyde dehydrogenase and suppresses ethanol intake of Syrian golden hamsters. Proc. Natl. Acad. Sci. U. S. A. 94:1675-1679.

14. Staub, R.E., Quistad, G.B., and Casida, J.E. 1998. Mechanism for benomyl action as a mitochondrial aldehyde dehydrogenase inhibitor in mice. Chem. Res. Toxicol. 11:535-543.

15. Mulsch, A., et al. 2001. Effects of in vivo nitroglycerin treatment on activity and expression of the guanylyl cyclase and cGMP-dependent protein kinase and their downstream target vasodilator-stimulated phosphoprotein in aorta. Circulation. 103:2188-2194.

16. Schulz, E., et al. 2002. Functional and biochemical analysis of endothelial (dys)function and NO/cGMP signaling in human blood vessels with and without nitroglycerin pretreatment. Circulation. 105:1170-1175.

17. Brien, J.F., et al. 1988. Mechanism of glyceryl trinitrate-induced vasodilation. I. Relationship between drug biotransformation, tissue cyclic GMP elevation and relaxation of rabbit aorta. J. Pharmacol. Exp. Ther. 244:322-327.

18. Hellstrom-Lindahl, E., and Weiner, H. 1985. Effects of disulfiram on the oxidation of benzaldehyde and acetaldehyde in rat liver. Biochem. Pharmacol. 34:1529-1535.

19. Raha, S., McEachern, G.E., Myint, A.T., and Robinson, B.H. 2000. Superoxides from mitochondrial complex III: the role of manganese superoxide dismutase. Free Radic. Biol. Med. 29:170-180.

20. Nishinaka, Y., et al. 1993. A new sensitive chemiluminescence probe, L-012, for measuring the production of superoxide anion by cells. Biochem. Biophys. Res. Commun. 193:554-559.

21. Sohn, H.Y., Gloe, T., Keller, M., Schoenafinger, K., and Pohl, U. 1999. Sensitive superoxide detection in vascular cells by the new chemiluminescence dye L-012. J. Vasc. Res. 36:456-464.

22. King, M.P., and Attardi, G. 1996. Isolation of human cell lines lacking mitochondrial DNA. Methods Enzymol. 264:304-313.

23. Nemoto, S., Takeda, K., Yu, Z.X., Ferrans, V.J., and Finkel, T. 2000. Role for mitochondrial oxidants as regulators of cellular metabolism. Mol. Cell. Biol. 20:7311-7318

24. Huang, A., Vita, J.A., Venema, R.C., and Keaney, J.F., Jr. 2000. Ascorbic acid enhances endothelial nitric-oxide synthase activity by increasing intracellular tetrahydrobiopterin. J. Biol. Chem. 275:17399-17406.

25. Jugdutt, B.I., and Warnica, J.W. 1989. Tolerance with low dose intravenous nitroglycerin therapy in acute myocardial infarction. Am. J. Cardiol. 64:581-587.

26. Elkayam, U., et al. 1987. Incidence of early tolerance to hemodynamic effects of continuous infusion of nitroglycerin in patients with coronary artery disease and heart failure. Circulation. 76:577-584.

27. Gori, T., et al. 2001. Folic acid prevents nitroglycerin-induced nitric oxide synthase dysfunction and nitrate tolerance: a human in vivo study. Circulation. 104:1119-1123. 
28. Gori, T., Mak, S.S., Kelly, S., and Parker, J.D. 2001. Evidence supporting abnormalities in nitric oxide synthase function induced by nitroglycerin in humans. J. Am. Coll. Cardiol. 38:1096-1101.

29. Heitzer, T., Schlinzig, T., Krohn, K., Meinertz, T., and Munzel, T. 2001 Endothelial dysfunction, oxidative stress, and risk of cardiovascular events in patients with coronary artery disease. Circulation. 104:2673-2678

30. Oelze, M., et al. 2000. Vasodilator-stimulated phosphoprotein serine 239 phosphorylation as a sensitive monitor of defective nitric oxide/cGMP signaling and endothelial dysfunction. Circ. Res. 87:999-1005.

31. Warnholtz, A., Tsilimingas, N., Wendt, M., and Munzel, T. 2002. Mechanisms underlying nitrate-induced endothelial dysfunction: insight from experimental and clinical studies. Heart Fail. Rev. 7:335-345.

32. Caramori, P.R., et al. 1998. Therapy with nitroglycerin increases coronary vasoconstriction in response to acetylcholine. J. Am. Coll. Cardiol. 32:1969-1974.

33. Bassenge, E., Fink, N., Skatchkov, M., and Fink, B. 1998. Dietary supplement with vitamin $C$ prevents nitrate tolerance. J. Clin. Invest. 102:67-71.
34. Warnholtz, A., et al. 2002. Adverse effects of nitroglycerin treatment on endothelial function, vascular nitrotyrosine levels and cGMP-dependent protein kinase activity in hyperlipidemic Watanabe rabbits. J. Am. Coll. Cardiol. 40:1356-1363.

35. Skatchkov, M., Larina, L.L., Larin, A.A., Fink, N., and Bassenge, E. 1997. Urinary nitrotyrosine content as a marker of peroxynitrite-induced tolerance to organic nitrates. J. Cardiovasc. Pharmacol. Ther. 2:85-96.

36. Needleman, P., and Hunter, F.E., Jr. 1966. Effects of organic nitrates on mitochondrial respiration and swelling: possible correlations with the mechanism of pharmacologic action. Mol. Pharmacol. 2:134-143.

37. Needleman, P., and Hunter, F.E., Jr. 1965. The transformation of glyceryl trinitrate and other nitrates by glutathione-organic nitrate reductase. Mol Pharmacol. 1:77-86.

38. Nakamura, Y., Moss, A.J., Brown, M.W., Kinoshita, M., and Kawai, C. 1999. Long-term nitrate use may be deleterious in ischemic heart disease: a study using the databases from two large-scale postinfarction studies. Multicenter Myocardial Ischemia Research Group. Am. Heart J. 138:577-585 